\title{
ON SOME 4-DIMENSIONAL ALMOST KÄHLER MANIFOLDS
}

\author{
Tedi C. Draghici
}

\section{Introduction}

The following conjecture of Goldberg is still open: The almost complex structure of a compact, almost Kähler Einstein manifold is integrable (and therefore the manifold is Kähler).

Progress has been made by K. Sekıgawa who proved that if the scalar curvature is nonnegative then the conjecture is true $([8,9])$.

In 1986, studying variations in the set of metrics associated to a given symplectic form on a compact, symplectic manifold, Blair and Ianus showed that the commutativity of the Ricci operator with the almost complex structure is the critical point condition for a certain class of Riemannian functionals ([1]). They asked a question stronger than Goldberg: For a compact almost Kähler manifold whose Ricci operator commutes with the almost complex structure, is it true that the almost complex structure must be integrable? In [2] Davidov and Muskarov gave an example showing that the answer to the latter question is negative. However, it is still interesting to see under what additional assumptions we have a positive answer to the question of Blair and Ianus. Also, the example given by Davidov and Muskarov is 6-dimensional and it might be possible that in dimension 4 the situation is different.

It is well known that on any almost Hermitian manifold we can define the *-Ricci tensor, which is an analogue of the Ricci tensor but involving also the almost complex structure. On a Kähler manifold the Ricci and the *-Ricci tensors coincide. For this reason the problem of a star version of Goldberg conjecture has been raised, replacing the Einstein condition with one on the *-Ricci tensor.

The purpose of this note is to study some aspects of these questions in dimension 4.

I am deeply grateful to Professor David E. Blair, who constantly guided and encouraged me while working on this paper.

Recerved November 8, 1993. 


\section{Preliminaries}

Let $M=(M, J, g)$ be a 4-dimensional, compact, almost Hermitian manifold. We assume that $M$ is oriented by the volume form $\sigma=(1 / 2) \Omega^{2}$, where $\Omega$ is the Kähler form defined by $\Omega(X, Y)=g(X, J Y)$ for any $X, Y$ vector fields on $M$. By the well known process of raising and lowering indices, the metric $g$ induces a scalar product in each fiber of $\boldsymbol{T}^{r, s} M$, the fiber bundle of tensors of type $(r, s)$ on $M$. We will denote this scalar product by $($,$) and the corresponding$ norm by $\|$. Integrating over the manifold we obtain a global scalar product

$$
\langle,\rangle=\int_{M}(,) \sigma .
$$

On $\Lambda^{2} M$, the fiber bundle of 2 -forms on $M$, the induced scalar product in each fiber is defined by:

$$
(\alpha, \beta)=\frac{1}{2} \sum_{\imath, j} \alpha_{\imath j} \beta_{\imath \jmath},
$$

where $\alpha, \beta \in \Lambda_{p}^{2} M$, for some point $p \in M ; \alpha_{\imath \jmath}=\alpha\left(e_{2}, e_{\jmath}\right),\left\{e_{1}, e_{2}, e_{3}, e_{4}\right\}$ being an orthonormal basis of the tangent space at $p, \boldsymbol{T}_{p} M$.

It is well known that on a 4-dimensional Riemannian manifold the Hodge operator acting on 2 -forms has the property $\star^{2}=$ id and hence induces the decomposition :

$$
\Lambda^{2} M=\Lambda_{+}^{2} M \oplus \Lambda_{-}^{2} M,
$$

where $\Lambda_{+}^{2} M$ and $\Lambda_{-}^{2} M$ denote the sub-bundles of self-dual 2 -forms and anti selfdual 2 -forms respectively. This decomposition is orthogonal in respect to the scalar product (, ). Also, it is easily checked that $(\alpha, \beta) \sigma=\alpha \wedge \star \beta$.

On a 4-dimensional almost Hermitian manifold another decomposition of $\Lambda^{2} M$ holds (see for example [8]):

$$
\Lambda^{2} M=\boldsymbol{R} \Omega \oplus \Lambda_{0}^{(1,1)} M \oplus \boldsymbol{L} M,
$$

where $\Lambda_{0}^{(1,1)} M$ denotes the vector bundle of real, J-invariant two-forms with vanishinng trace (for the trace of a 2 -form we adopt the convention: $\operatorname{tr}(\alpha)=$ $1 / 2(\alpha, \Omega)$ ), and $L M$ the vector bundle of real J-anti-invariant 2 -forms on $M$. This decomposition is also orthogonal in respect to (, ) and, moreover, we have

$$
\Lambda_{+}^{2} M=\boldsymbol{R} \Omega \oplus \boldsymbol{L} \boldsymbol{M}, \quad \Lambda_{-}^{2} M=\Lambda_{0}^{(1,1)} M .
$$

If $\phi$ is any two-form on $M$ we can therefore write:

$$
\phi=\operatorname{tr} \psi \Omega+\phi_{0}^{(1,1)}+\phi^{a n t \imath},
$$

where $\operatorname{tr} \psi \Omega+\psi^{a n t \imath}$ is the self dual part and $\phi_{0}^{(1,1)}$ is the anti-self dual part of the form. Using this we get 


$$
\psi \wedge \psi=\psi \wedge\left[*\left(\operatorname{tr} \psi \Omega+\psi^{a n t \imath}\right)-* \psi_{0}^{(1,1)}\right]=\left(\phi, \operatorname{tr} \psi \Omega+\psi^{a n t \imath}-\psi_{0}^{(1,1)}\right) \sigma .
$$

Therefore, on a 4-dimensional almost Hermitian manifold, for any 2-form $\phi$ we have

$$
\phi^{2}=\left(2 \operatorname{tr}^{2} \psi-\left|\phi_{0}^{(1,1)}\right|^{2}+\left|\phi^{a n t \imath}\right|^{2}\right) \sigma .
$$

This formula was used by Gauduchon for Hermitian 4-dimensional manifolds (see [4]).

We recall that an almost Hermitian manifold whose Kähler form $\Omega$ is closed, is called an almost Kähler manifold. A consequence of $d \Omega=0$ is the following equality:

$$
\left(\nabla_{J X} J\right) J Y=-\left(\nabla_{X} J\right) Y
$$

or equivalently

$$
\left(\nabla_{J X} \Omega\right)(J Y, Z)=-\left(\nabla_{X} \Omega\right)(Y, Z)
$$

for any tangent vectors $X, Y, Z$. An almost Hermitian manifold satisfying just (3) (or (4)) is called quasi-Kähler and there are known examples of quasi-Kähler manifolds which are not almost Kähler. In dimension 4 however, every quasiKähler manifold is almost Kähler. After all, the condition that the Kähler form is closed is equivalent to

$$
\left(\nabla_{X} \Omega\right)(Y, Z)+\left(\nabla_{Y} \Omega\right)(Z, X)+\left(\nabla_{Z} \Omega\right)(X, Y)=0,
$$

for $X, Y, Z \in \mathscr{X}(M)$. Since the left hand-side is tensorial in $X, Y, Z$, it is enough to check the equality only for a preferred basis of tangent vectors. For any $p \in M$, we can choose in a neighborhood of $p$ a $\mathrm{J}$-basis of tangent vector fields, that is, an orthonormal basis of the form $\left\{e_{1}, J e_{1}, e_{2}, J e_{2}\right\}$. The quasi-Kähler condition (4) and the fact the skew-symmetry is preserved by taking covariant derivatives imply that (5) holds if $X, Y, Z \in\left\{e_{1}, J e_{1}, e_{2}, J e_{2}\right\}$ and, therefore, (5) holds for any $X, Y, Z$.

Another formula which holds on any quasi-Kähler manıfold and we will often use is

$$
\Sigma\left(\nabla_{e_{i}} J\right) e_{\imath}=0 .
$$

This is an easy consequence of (3) and is just the local expression of the fact that $\Omega$ is co-closed. Therefore on any almost Kähler manifold the Kähler form $\Omega$ is harmonic, being closed and co-closed.

If $(M, g, J)$ is an almost Hermitian manifold and $D$ a $(2,0)$ tensor on $M$, we say that $D$ is $J$-invarnant (or $J$-Hermitian) if it satisfies

$$
D(J X, J Y)=D(X, Y)
$$

for any tangent vectors $X, Y$.

We say that $D$ is $J$-anti invarıant (or $J$-antı Hermitian) if it satisfies

$$
D(J X, J Y)=-D(X, Y)
$$


for any tangent vectors $X, Y$.

Let us recall now a definition due to Gray ([6]). For every point $p \in M$, he defines $\mathscr{D}_{p}:=\left\{X \in\left\{T_{p} M \mid \nabla_{X} J=0\right\}\right.$ and calls $\mathscr{D}$ the Kähler nullity distribution of the almost Hermitian manifold $M$. Note that $\mathscr{D}$ need not be a distribution in the usual sence the dimension might vary with the point.

From now on, let $(M, g, J, \Omega)$ be a 4-dimensional almost Kähler manifold.

It follows from relation (4) that $\nabla_{X} \Omega$ belongs to the sub-fibration $L M$ of $\Lambda^{2} M$, for any vector $X$. Also, $\nabla_{X} \Omega=0$ if and only if $\nabla_{J X} \Omega=0$. Therefore we conclude that $\operatorname{dim} \mathscr{D}_{p}$ is an even number.

Now, since $M$ is 4 -dimensional, the fibers of $\boldsymbol{L} M$ have dimension 2. Therefore, locally we can write

$$
\nabla \Omega=\alpha \otimes \Phi+\beta \otimes J \Phi
$$

where $\{\Phi, J \Phi\}$ is any (local) orthonormal frame of $L M$, and $\alpha, \beta$ are (local) 1 -forms (see [8]). Hence, $\mathscr{D}_{p}$ contains the intersection of $\operatorname{ker} \alpha_{p}$ and $\operatorname{ker} \beta_{p}$. This proves that the Kähler nullity distribution $\mathscr{D}_{p}$ has dimension either 2 or 4 .

Let us consider now the following $(2,0)$ tensor field:

$$
D(X, Y)=2\left(\nabla_{X} \Omega, \nabla_{Y} \Omega\right),
$$

where (,) is the local scalar product introduced above. $D$ is obviously symmetric, and, from the quasi-Kähler condition (4), we obtain that it is also Jinvariant. Thus, at every point, $D$ has two double eigenvalues $\lambda_{1}, \lambda_{2}$, or $D=0$. Since we have shown that $\mathscr{D}_{p}$ has dimension at least 2 , one of the eigenvalues, say $\lambda_{1}$, is 0 . As $\operatorname{tr} D=|\nabla J|^{2}$, it follows that $\lambda_{2}=(1 / 2)|\nabla J|^{2}$. We will need this in the next section.

To complete this section we introduce some additional notation which we will need later. $(M, g, J, \Omega)$ will be an almost Kähler manifold, compact and 4-dimensional. We denote by $\nabla, R, \rho, \tau$ the Levi-Civita connection, the Riemannian curvature tensor, the Ricci tensor and the scalar curvature of $M$ respectively. Our conventions for the definitions of the curvature and the Ricci tensor are the following:

$$
\begin{gathered}
R(X, Y)=\left[\nabla_{X}, \nabla_{Y}\right]-\nabla_{[X, Y]}, \\
\rho(X, Y)=\operatorname{tr}(Z \longrightarrow R(Z, X) Y) .
\end{gathered}
$$

We also denote by $\rho^{\star}$ and $\tau^{\star}$ the star-Ricci tensor and the star-scalar curvature of $M$ respectively (cf. [1], [8], [9]).

It is well known that the Ricci tensor is symmetric, whereas, in general, the star-Ricci tensor is neither symmetric nor skew-symmetric. We may remark though, that the star-Ricci tensor satisfies the following identity:

$$
\rho^{\star}(J X, J Y)=\rho^{\star}(Y, X),
$$


for any tangent vectors $X, Y$. This implies that the symmetric part of the star-Ricci tensor, denoted by $\rho^{\star s y m}$, is a J-invariant tensor, i. e. it satisfies

$$
\rho^{\star s y m}(J X, J Y)=\rho^{\star s y m}(X, Y),
$$

while the skew-symmetric part, denoted by $\rho^{\star s k e w}$, is a J-anti-invariant tensor, i. e.

$$
\rho^{\star s k e w}(J X, J Y)=-\rho^{\star s k e w}(X, Y) .
$$

In general, the Ricc1 tensor does not have any special behavior in respect to $J$, and we will often use the decomposition

$$
\rho=\rho^{\imath n v}+\rho^{a n t \imath},
$$

where $\rho^{\imath n v}$ in the J-invariant part of the Ricci tensor defined by

$$
\rho^{\imath n v}(X, Y)=\frac{1}{2}(\rho(X, Y)+\rho(J X, J Y)),
$$

and $\rho^{a n t \imath}$ is the J-anti invariant part given by

$$
\rho^{a n t \imath}(X, Y)=\frac{1}{2}(\rho(X, Y)-\rho(J X, J Y)) .
$$

For any 4-dimensional, almost Hermitian manifold, Tricceri and Vanhecke ([12]) showed that

$$
\rho^{\imath n v}-\rho^{\star s y m}=\frac{1}{4}\left(\tau-\tau^{\star}\right) g
$$

and also

$$
R(J X, J Y, J Z, J W)=R(X, Y, Z, W) \Longleftrightarrow\left(\rho=\rho^{\imath n v}, \rho^{\star}=\rho^{\star s y m}\right) .
$$

If the Ricci tensor is at every point a multiple of the metric we say that the manifold is Einstein. Note that in this case it follows that the scalar curvature is a constant, so the Ricci tensor is a constant multiple of the metric. Such a result is not true for the *-Ricci tensor and this leads to two different versions of the $*$-Einstein condition. The term weakly *-Einstein manifold is now commonly used when the *-Ricci tensor is at every point a multiple of the metric, keeping the name *-Einstern manifold for the case when the $*$-Ricci tensor is a constant multiple of the metric (see [10], [11]). We will make this distinction throughout the paper.

We will also use the following known formula of the first Chern form, valid for any almost Hermitian manifold:

$$
8 \pi \gamma_{\imath \jmath}=-4 \rho_{i j}^{\star_{j}}+\left(\nabla_{\imath} J_{\bar{k} h}\right)\left(\nabla_{\jmath} J_{k h}\right) .
$$

If not otherwise specified, our local computations and formulas are written in a local J-basis, 1. e. an orthonormal basis of the form $\left\{e_{1}, e_{2}, J e_{1}, J e_{2}\right\}$. Thus $\rho_{\imath j}^{\star}$ means $\rho^{\star}\left(e_{\imath}, J e_{j}\right)$. The indices $\imath$,, will run from 1 to 4 , with the conven- 
tion that $e_{3}, e_{4}$ are respectively $J e_{1}, J e_{2}$. We adopt the summation convention of Einstein, but, as we work with orthonormal bases, there is no need to raise and lower the indices.

\section{Some Results}

As we mentioned before, Sekigawa proved that a compact Einstein, almost Kähler manifold with nonnegative scalar curvature is Kähler. The key of the proof is to establish first an inequality which holds on any compact, almost Kähler manifold.

Proposition 1 (Sekigawa). Let $(M, J, g, \Omega)$ be a $2 n$-dimensional $(n \geqq 2)$ compact almost Kähler manifold. Then

$$
\begin{aligned}
0 \leqq & -2 \int_{M}\left[\sum\left(\nabla_{i} \rho_{b j}-\nabla_{j} \rho_{b \imath}\right)\left(\nabla_{b} J_{\imath k}\right) J_{j k}+\sum \rho_{i j}\left(\nabla_{b} J_{i k}\right)\left(\nabla_{b} J_{j k}\right)\right] \sigma- \\
& -\frac{1}{2 n} \int_{M}|\nabla J|^{4} \sigma .
\end{aligned}
$$

For the proof we refer to [9]. It can be easily obtained from Lemmas 2.2, 2.5, 2.7 and Proposition 3.2 of that paper. Now if $M$ is Einstein, $\nabla \rho=0$ and

$$
\sum \rho_{i j}\left(\nabla_{b} J_{\imath k}\right)\left(\nabla_{b} J_{j k}\right)=\frac{\tau}{n}|\nabla J|^{2},
$$

so the right-hand side of (10) is nonpositive. Therefore, all the terms in (10) must vanish, in particular, $\nabla J=0$.

This is the sketch for the proof Sekigawa gave to his result.

Starting with this inequality, not assuming the Einstein condition, but only that the Ricci tensor is J-invariant, we have to work a little bit more to get some conclusions.

First we use integration by parts and equation (6) to get

$$
\begin{aligned}
& \int_{M}\left[\sum\left(\nabla_{i} \rho_{b j}-\nabla_{j} \rho_{b \imath}\right)\left(\nabla_{b} J_{\imath k}\right) J_{j k}\right] \sigma \\
& =\int_{M}\left[\sum \rho_{b \imath} \nabla_{j}\left(\nabla_{b} J_{i k}\right) J_{j k}-\sum \rho_{b j} \nabla_{i}\left(\left(\nabla_{b} J_{i k}\right) J_{j k}\right)\right] \sigma .
\end{aligned}
$$

Here let us remark that since $J^{2}=-I d$, we have

$$
\left(\nabla_{b} J_{i k}\right) J_{j k}=-J_{i k}\left(\nabla_{b} J_{j k}\right),
$$

which implies, by virtue of (6) that

$$
\nabla_{i}\left(\left(\nabla_{b} J_{i k}\right) J_{j k}\right)=-J_{i k} \nabla_{i}\left(\nabla_{b} J_{j k}\right) .
$$

Therefore we get 


$$
\int_{M}\left[\left(\nabla_{i} \rho_{b j}-\nabla_{j} \rho_{b i}\right)\left(\nabla_{b} J_{\imath k} \sum J_{j k}\right] \sigma=2 \int_{M} \sum \rho_{b i} \nabla_{j}\left(\nabla_{b} J_{i k}\right) J_{j k} \sigma .\right.
$$

On any quasi-Kähler manifold we have the following identity which can be easily obtained after a straightforward computation making use of (3):

$$
\nabla_{j} \nabla_{\bar{b}} J_{i_{k}}=-\nabla_{j} \nabla_{b} J_{i k}-\left(\nabla_{j} J_{b s}\right)\left(\nabla_{s} J_{\bar{i}_{k}}\right)-\left(\nabla_{\bar{b}} J_{s k}\right)\left(\nabla_{j} J_{i s}\right) .
$$

Since $\rho$ is J-invariant, using the formula above and, repeatedly, the quasi-Kähler condition (3) we obtain :

$$
\rho_{b i}\left(\nabla_{j} \nabla_{b} J_{i k}\right) J_{j k}=\frac{1}{2} \rho_{b i}\left(\nabla_{k} J_{b s}\right)\left(\nabla_{s} J_{\imath k}\right)-\frac{1}{2} \rho_{b i}\left(\nabla_{b} J_{k s}\right)\left(\nabla_{k} J_{i s}\right) .
$$

Multiplying by 2 and using several times equation (5) we get

$$
2 \rho_{b i}\left(\nabla_{j} \nabla_{b} J_{i k}\right) J_{j k}=\rho_{b i}\left(\nabla_{s} J_{b k}\right)\left(\nabla_{s} J_{i k}\right)-\rho_{b i}\left(\nabla_{b} J_{s k}\right)\left(\nabla_{\imath} J_{s k}\right) .
$$

After these computations inequality (10) becomes:

$$
0 \leqq-2 \int_{M}\left[2 \rho_{b i}\left(\nabla_{s} J_{b k}\right)\left(\nabla_{s} J_{i k}\right)-\rho_{b i}\left(\nabla_{b} J_{s k}\right)\left(\nabla_{\imath} J_{s k}\right)\right] \sigma-\frac{1}{2 n} \int_{M}|\nabla J|^{4} \sigma .
$$

The following result was proved in [3].

THEOREM 1. Let $M$ be a 2n-dimensional compact almost Kähler manifold, whose Ricci tensor is J-Hermitian and suppose there exists $\lambda \geqq 0$ such that $\lambda g(X, X)$ $\leqq \rho(X, X) \leqq 2 \lambda g(X, X)$ for any $X \in T M$. Then the almost complex structure is integrable, that is, $M$ is a Kähler manifold.

If $0 \leqq \lambda_{1} \leqq \cdots \leqq \lambda_{n}$ are the eigenvalues of $\rho$ (each of them is a double eigenvalue since $\rho$ is J-invariant), the "pinching" condition on the Ricci tensor insures that we have the inequality $2 \lambda_{1} \geqq \lambda_{n}$, so our metric is not "too far" from an Einstein one. In dimension 4 the result is even better. We don't have to assume anything about the eigenvalues of $\rho$, but only to be nonnegative. Hence the result of Sekigawa concerning Goldberg conjecture fully extends in dimension 4 to the problem of Blair and Ianus.

THEOREM 2. A 4-dimensional, compact, almost Kähler manifold, with Hermitian and nonnegative definite Ricci tensor is Kähler.

Proof. Let us denote by $B$ the symmetric, J-invariant tensor given by $B_{\imath \jmath}=\left(\nabla_{s} J_{t_{\imath}}\right)\left(\nabla_{s} J_{t j}\right)$. We can see that $B=(1 / 4)|\nabla J|^{2} g$ as follows.

For any almost Kähler manifold we have the following formula due to Koto $([7])$ :

$$
\rho^{\star s y m}=\rho^{2 n v}+\frac{1}{2} B
$$


This formula implies the well known relation between the scalar curvatures:

$$
\tau^{\star}=\tau+\frac{1}{2}|\nabla J|^{2}
$$

But in dimension 4 , we have the relation (7) between the Ricci tensors. Therefore, by (12), (13) and (7), $B=(1 / 4)|\nabla J|{ }^{2} g$.

Using this, one of the terms in (11) has a simpler expression:

$$
2 \rho_{b i}\left(\nabla_{s} J_{b k}\right)\left(\nabla_{s} J_{i k}\right)=\frac{\tau}{2}|\nabla J|^{2} .
$$

Now we choose a basis $\left\{e_{1}, J e_{1}, e_{2}, J e_{2}\right\}$ which diagonalizes the tensor $D$ considered in the previous section. In local coordinates $D_{b i}=\left(\nabla_{b} J_{s k}\right)\left(\nabla_{\imath} J_{s k}\right)$. As we remarked in Section 2, this tensor is symmetric, J-invariant, has a double eigenvalue 0 and another double eigenvalue $\lambda=(1 / 2)|\nabla J|^{2}$. Suppose $e_{1}, J e_{1}$ are eigenvectors corresponding to the eigenvalue 0 and $e_{2}, J e_{2}$ are eigenvectors corresponding to the eigenvalue $\lambda$. In this basis

$$
\rho_{b i}\left(\nabla_{b} J_{s k}\right)\left(\nabla_{\imath} J_{s k}\right)=\rho_{22}|\nabla J|^{2},
$$

and, since $\tau=2\left(\rho_{11}+\rho_{22}\right)$,

$$
2 \rho_{b j}\left(\nabla_{s} J_{b k}\right)\left(\nabla_{s} J_{i k}\right)-\rho_{b i}\left(\nabla_{b} J_{s k}\right)\left(\nabla_{\imath} J_{s k}\right)=\rho_{11}|\nabla J|^{2} .
$$

By hypothesis, the Ricci tensor is nonnegative definite, so $\rho_{11}=\rho\left(e_{1}, e_{1}\right) \geqq 0$, hence

$$
2 \rho_{b i}\left(\nabla_{s} J_{b k}\right)\left(\nabla_{s} J_{i k}\right) \geqq \rho_{b i}\left(\nabla_{b} J_{s k}\right)\left(\nabla_{\imath} J_{s k}\right) .
$$

Now, inequality (11) implies $|\nabla J|=0$, hence $J$ is parallel, so the manifold is Kähler.

If Goldberg conjecture is true (or its star version), one way to prove it might be the following: first show that on any compact symplectic manifold with the first Chern class a multiple of $[\Omega]$, an associated Einstein metric (if exists) must be Kähler, and then, the second step, show that on all other compact symplectic manifolds there are no Einstein associated metrics. Let us remark that the condition that the first Chern class is a multiple of $[\Omega]$ has been studied in two different contexts. In symplectic topology, symplectic manifolds satisfying this condition are called monotone, whereas in Kähler geometry, a Kähler manifold with $c_{1}(M)=\lambda[\Omega]$ is known as a cohomology Einstein Kähler manifold.

Here we prove the following proposition:

Proposition 2. Let $M$ be a compact, symplectic, 4-dimensional manifold with $c_{1}(M)=(1 / 8 \pi) \lambda[\Omega]$, for $\lambda \in \boldsymbol{R}$. Suppose there exist a weakly *-Einstern associated metric with the property that there exist three real numbers $a, b, c$ $\left(a \geqq 0, b \geqq 0\right.$, but $\left.a^{2}+b^{2} \neq 0\right)$ such that 


$$
a \tau+b \tau^{*}=c .
$$

Then this must be a Kähler metrac.

Proof. Without loss of generality we can assume that the total volume of $M$ is 1 .

If $\gamma$ is the first Chern form, then, from our assumptions, there exist a 1 form $\alpha$ such that

$$
8 \pi \gamma=\lambda \Omega+d \alpha .
$$

As $\Omega$ is harmonic $\langle\Omega, d \alpha\rangle=0$, so by (9) and the previous relation we get

$$
\lambda=-\frac{1}{2} \int_{M}\left(\tau+\tau^{\star}\right) \sigma
$$

Also, taking wedge product,

Therefore we get

$$
(8 \pi \gamma)^{2}=\lambda^{2} \Omega^{2}+d \alpha^{\prime}
$$

$$
c_{1}^{2}(M)=\frac{1}{32 \pi^{2}} \lambda^{2} .
$$

On the other hand, using the decomposition (1) and the formula (2) for $\phi=$ $8 \pi \gamma$ we get

$$
c_{1}^{2}(M)=\frac{1}{16 \pi^{2}} \int_{M}\left[\left(\tau^{\star}\right)^{2}-2\left|\rho^{\star s y m}\right|^{2}+2\left|\rho^{\star s k e w}\right|^{2}-\frac{1}{4}\left(\tau^{\star}+\tau\right)|\nabla J|^{2}+(\rho, D)\right] \sigma,
$$

where all the norms on the right hand-side are thought of as norms of tensors, so the $1 / 2$ coefficient from the local scalar product of forms does not appear here. The above formula is the almost Kähler case of the general expression of $c_{1}^{2}(M)$ obtained by Sekigawa in [8] for any 4-dimensional almost Hermitian manifold.

Comparing the two expressions for $c_{1}^{2}(M)$ we have

$$
\frac{1}{2} \lambda^{2}=\int_{M}\left[\left(\tau^{\star}\right)^{2}-2\left|\rho^{\star s y m}\right|^{2}+2\left|\rho^{\star s k e w}\right|^{2}-\frac{1}{4}\left(\tau^{\star}+\tau\right)|\nabla J|^{2}+(\rho, D)\right] \sigma .
$$

Working with the right-hand side, using (7) and (13), the previous equation becomes :

$$
\frac{1}{2}\left(\int_{M} \frac{\tau^{\star}+\tau}{2} \sigma\right)^{2}=\int_{M}\left[\tau^{2}-2\left|\rho^{\imath n v}\right|^{2}+2\left|\rho^{\star s k e w}\right|^{2}+(\rho, D)\right] \sigma .
$$

The weak *-Einstein condition means that $\rho^{\star s k e w}=0$ and $\rho^{\star s y m}=(1 / 4) \tau^{\star} g$. From (7) we deduce also that $\rho^{2 n v}=(1 / 4) \tau g$. With these relations, (14) becomes

$$
\left(\int_{M} \frac{\tau^{\star}+\tau}{2} \sigma\right)^{2}=\int_{M} \tau \tau^{\star} \sigma
$$

which we rewrite as 


$$
\left(\int_{M} \frac{\tau^{\star}-\tau}{2} \sigma\right)^{2}=\int_{M} \tau \tau^{\star} \sigma-\left(\int_{M} \tau \sigma\right)\left(\int_{M} \tau^{\star} \sigma\right)
$$

Suppose that $a \tau+b \tau^{\star}=c$, with $a, b, c$ constants, $a, b \geqq 0$, but not simultaneously 0 . Without loss of generality we can assume $b \neq 0$. Then

$$
\int_{M} \tau \tau^{\star} \sigma-\left(\int_{M} \tau \sigma\right)\left(\int_{M} \tau^{\star} \sigma\right)=\frac{a}{b}\left[\left(\int_{M} \tau \sigma\right)^{2}-\int_{M} \tau^{2} \sigma\right]
$$

By Cauchy-Schwarz inequality we see that this last expression is nonpositive. Hence the right hand-side of (16) is nonpositive, whereas the left hand-side is nonnegative. This implies that $\tau^{\star}=\tau$ and, by (13), the metric is Kähler.

Without any effort, we have the following corollaries:

COROLlARY 1. Let $M$ be a compact, symplectıc, 4-dimensional manifold with $c_{1}(M)=(1 / 8 \pi) \lambda[\Omega]$, for $\lambda \in \boldsymbol{R}$. Any $\star$-Einstein associated metric is a Kähler metric.

COROLlaRy 2. Let $M$ be a compact, symplectic, 4-dimensional manifold with $c_{1}(M)=(1 / 8 \pi) \lambda[\Omega]$, for $\lambda \in \boldsymbol{R}$. Any Einstern, weakly $\star$-Einstein associated metruc is a Kähler metric.

Since, in general, on an almost Kähler manifold the Ricci tensor is not Jinvariant we cannot speak of a Ricci form as in the Kähler case. We can try to define a Ricci form on any almost Kähler manifold by

$$
\alpha(X, Y)=\rho^{2 n v}(X, J Y) .
$$

The following Proposition was proved in [3]:

Proposition 3. Let $(M, g, J, \Omega)$ be a compact almost Kähler manifold such that the Ricci form $\alpha$ belongs to the cohomology class $2 \pi c_{1}(M)$, where $c_{1}(M)$ is the first Chern class of the manifold. Then the mantfold is Kähler.

It seems that we are assuming too much, asking at the same time that the Ricci form $\alpha$ be closed and in the cohomology class $2 \pi c_{1}(M)$. In dimension 4 the situation is better.

Proposition 4. On a compact, 4-dimensional almost Kähler manifold with J-invariant Ricci tensor, the Ricct form is closed.

Let us remark first that since $\rho=\rho^{\imath n v}$, the Ricci form we introduced, $\alpha$, is now exactly what it should be in the Kähler case.

For the proof of the Proposition we need the following Lemma:

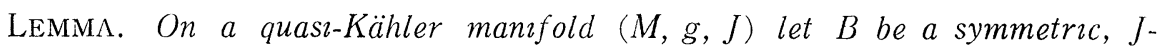


invariant tensor field of type $(2,0)$, satisfyng $\delta B=0$. Then the 2-form $\beta(X, Y)$ $=B(X, J Y)$ is co-closed (i.e. $\delta \beta=0$ ).

Proof of Lemma. We work in a local J-basis $\left\{e_{1}, \cdots, e_{n}, J e_{1}, \cdots, J e_{n}\right\}$. Locally, $\beta$ is given by $\beta_{\imath \jmath}=B_{i s} J_{\jmath s}$. Since $\nabla_{i} B_{i s}=0$, we have

$$
\nabla_{i} \beta_{\imath \jmath}=B_{\imath s} \nabla_{\imath} J_{\jmath s} .
$$

Using the J-invariance of $B$ and the quasi-Kähler condition (3) we see that

$$
B_{\imath s} \nabla_{\imath} J_{\jmath s}=B_{\bar{i} \bar{s}} \nabla_{i} J_{j \bar{s}}=B_{i s} \nabla_{\bar{\imath}} J_{j \bar{s}}=-B_{i s} \nabla_{\imath} J_{\jmath s} .
$$

Hence

$$
\nabla_{i} \beta_{\imath \jmath}=B_{\imath s} \nabla_{\imath} J_{\jmath s}=0
$$

Proof of Proposition 4. We apply the previous Lemma to the tensor field $B=\rho-(1 / 2) \tau g$. It is symmetric, J-invariant from the hypothesis and by the second Bianchi identity, $\delta B=0$. The corresponding 2 -form, $\beta=\alpha-(1 / 2) \tau \Omega$ is therefore co-closed. Since the co-differential operator on forms is given by $\delta=$ $-\star d \star, \star \beta$ is closed. Using the decomposition (1),

$$
\star \beta=\star\left(\alpha-\frac{1}{4} \tau g-\frac{1}{4} \tau g\right)=-\alpha+\frac{1}{4} \tau g-\frac{1}{4} \tau g=-\alpha .
$$

Thus $\alpha$ is closed.

Finally, we have the following result:

THEOREM 3. Let $(M, \Omega)$ be a compact, 4-dimensional symplectic manifold with $H^{2}(M ; \boldsymbol{R})=\boldsymbol{R}$. If there exists an associated almost Kähler structure $(M, g$, $J, \Omega$ ) such that its Racci tensor is J-invariant and its star Ricce tensor is symmetric, then this must be a Kähler structure.

Proof. Without loss of generality we assume again that the volume of $M$ is 1 .

Since $H^{2}(M ; \boldsymbol{R})=\boldsymbol{R}$, there exist real numbers $\lambda, \mu$ such that $c_{1}(M)=$ $(1 / 8 \pi) \lambda[\Omega]$ and $[\alpha]=\mu[\Omega]$. Hence $8 \pi \gamma-\lambda \Omega$ and $\alpha-\mu \Omega$ are exact, so orthogonal to $\Omega$ with respect to $\langle$,$\rangle . From this we get$

$$
\lambda=-\frac{1}{2} \int_{M}\left(\tau+\tau^{\star}\right) \sigma, \quad \mu=\frac{1}{4} \int_{M} \tau \sigma .
$$

We are under the assumptions of Proposition 4 , so the 2 -form $\beta=\alpha-(1 / 2) \tau \Omega$ is co-closed, and hence orthogonal to $8 \pi \gamma-\lambda \Omega$ and $\alpha-\mu \Omega$. Expressing this, we get 


$$
\begin{gathered}
\int_{M}\left[-4|\rho|^{2}+2 \tau^{2}+(\rho, D)\right] \sigma=\frac{1}{2}\left(\int_{M} \tau \sigma\right)\left(\int_{M}\left(\tau^{\star}+\tau\right) \sigma\right) \\
\int_{M}|\rho|^{2} \sigma=\frac{1}{2} \int_{M} \tau^{2} \sigma-\frac{1}{4}\left(\int_{M} \tau \sigma\right)^{2}
\end{gathered}
$$

Relations (17) and (18) combined give

$$
\int_{M}(\rho, D) \sigma=\left(\int_{M} \tau \sigma\right)\left(\int_{M} \frac{\tau^{\star}-\tau}{2} \sigma\right)
$$

Using now (18) and (19) in (14), which holds under our assumption, we obtain

$$
\int_{M} 4\left|\rho^{\star s k e w}\right|^{2} \sigma=\left(\int_{M} \frac{\tau^{\star}-\tau}{2} \sigma\right)^{2}=\left(\int_{M} \frac{|\nabla J|^{2}}{4} \sigma\right)^{2} .
$$

By assumption the star Ricci tensor is symmetric, so (20) gives $\nabla J=0$ completing the proof.

It is interesting to compare Theorem 3 and the Corollaries of the Proposition 2 with the following result from $[10]$ :

Theorem (Sekigawa, Vanhecke). Let $M=(M, g, J)$ be a 4-dimensional compact, almost Kähler manifold which is Einstern and *-Einstein. Then $M$ is a Kähler manifold.

\section{REFERENCES}

[1] D. E. Blair and S. IAnUs, Critical associated metrics on symplectic manifolds, Contemp. Math., 51 (1986), 23-29.

[I J. Davidov And O. Muskarov, Twistor spaces with Hermitian Ricci tensor, Proc. Amer. Math. Soc., 109 (1990), 1115-1120.

[3] T. Draghici, On the almost Kähler manifolds with Hermitian Riccı tensor. Houston J. Math., 20 (1994), 293-298.

[4] P. Gauduchon, La 1-forme de torsion d'une variete hermitienne, Math. Ann., 267 (1984), 495-518.

[5] S.I. Goldberg, Integrability of almost Kähler manifolds, Proc. Amer. Math. Soc., 21 (1969), 96-100.

[6] A. GraY, Curvature identities for Hermitian and almost Hermitian manifolds, Tohoku Math. J., 28 (1976), 601-612.

[7] S. Koтo, Some theorems on almost Kählerian spaces, J. Math. Soc. Japan, 12 (1960), 422-433.

[8] K. Sekigawa, On some 4-dimensional compact almist Hermitian manifolds, J. Ramanujan Math. Soc., 2 (1987), 101-116.

[9] K. Sekigawa, On some compact Einstein almost Kähler manifolds, J. Math. Soc. Japan, 36 (1987), 677-684.

10 K. Sekigawa aNd L. Vanhecke, Four-dimensional almost Kähler Einstein manıfolds, Ann. Mat. Pura Appl. (4), 157 (1990), 149-160. 
[11] K. Sekigaiva and L. Vanhecke, Almost Hermitian manifolds with vanishing first Chern classes of Chern numbers, Rend. Sem. Mat. Univ. Politec. Torino, 50 (1992), 195-208.

[12] F. TRICCERI ANd L. VANHecke, Curvature tensors on almost Hermitian manifolds, Trans. Amer. Math. Soc., 267 (1981), 365-398.

Department of MATHEMATICS

Michigan State University

EAST LANSING, MI, 48824

U.S.A. 